\title{
Productivity and Management of Renewable Resources: Why More Efficient Fishing Fleets Should Fish Less
}

\author{
Eric Nævdal ${ }^{1}$ \\ Accepted: 25 November 2021 / Published online: 4 January 2022 \\ (C) The Author(s) 2021
}

\begin{abstract}
This article analyses the effect of productivity improvements on optimal fisheries management. It is shown that when harvest costs are independent of resource stock and the stock is below its steady state level, then for any given stock it is optimal to reduce harvest levels in response to a productivity increase unless optimal harvest rate is already zero. If harvest costs are stock dependent this result is modified; for stock dependent harvest costs there exists an interval of stock sizes below the steady state where it is optimal to reduce the harvest rate for any given stock size whereas if the harvest rate is close to an economically optimal steady state it is optimal to increase the harvest rate.
\end{abstract}

Keywords Fisheries · Optimal control $\cdot$ Productivity $\cdot$ Renewable resources

JEL Classification C61 · Q22

\section{Introduction}

Many of the world's fisheries are in dire straits because of poor management and poorly delineated property rights with a third of these fisheries labelled unsustainable by the Food and Agriculture Organization of the United Nations (FAO 2018). This poor state of affairs has been with us for some time and the costs are substantial (Clark 2005). The estimated global cost of forgone rents for the year 2012 alone was for instance estimated to be US\$ 83 billion for that year alone (World Bank 2017).

The problem of overfishing is likely to be exacerbated by technology. Brander and Taylor (1997) demonstrated that improved productivity could lead to more aggressive harvesting of open access resources. This also applies to open access fisheries and fisheries subject to the tragedy of the commons (Whitmarsh 1990; Squires and Vestergaard 2013; Squires and Vestergaard 2018). There are several well documented examples where improved technology has led to the collapse of a fish stock (Hannesson et al. 2010; Gordon and Hannesson 2015). Kvamsdal et al. (2016) point out that even in managed fisheries, “....increased technical efficiency and progress, usually lead to overcapacity in national fishing fleets.

Eric Nævdal

eric.navdal@frisch.uio.no

1 Frisch Centre, University of Oslo, Gaustadalléen 21, 0349 Oslo, Norway 
Overcapacity creates national pressure for higher quotas...". In a recent paper, Skonhoft and Quaas (2019) find that in a restricted open access or shared resource fishery if the long run steady state in a shared, but unmanaged, resource fishery is lower than the harvest rate associated with maximum sustainable yield, then improved technology may increase rents. However in larger, more accessible fisheries this condition is unlikely to hold.

The caveat in Skonhoft and Quaas (2019) not notwithstanding, it seems clear that improved technology worsens the market failure of a poorly managed fishery. This is unfortunate as improved technology has the potential to make the fishery more valuable and therefore makes it even more important that fish stocks are managed as well as possible and that the role of technology is understood. Remarkably little has been written on the role of technology in an optimally managed fishery. As stated in Squires and Vestergaard (2013): "... the normative literature has yet to formally analyze the impact of changes in disembodied and embodied technical progress and technical efficiency on optimum exploitation of common renewable resources."

The analysis in the literature on the effects of improved productivity is mostly concerned with steady state analysis and/or singular solutions resulting from bang-bang controls (Clark and Munro 1975; Caputo 1989; Squires and Vestergaard 2013, 2018). However steady state analysis is only locally relevant and policy advice for management of recovering stocks should not be based on steady state analysis. As we shall see, in fisheries such a strategy would be particularly ill advised as the effects of technology in the case of stock dependent costs go in completely different directions in steady state and in stock levels sufficiently below the optimal steady state. Squires and Vestergaard $(2013,2018)$ go some way in performing a formal analysis. Their contribution is contrasted with the results in the present paper in conjunction with the discussion of stock dependent cost functions.

The results are derived in general versions of the original canonical fisheries model (Clark and Munro 1975). The modelling of improved productivity is very basic and obtained by examining how an exogenous and unanticipated one-shot change in a productivity parameter affects optimal policy. This may seem restrictive. However, making the productivity change unanticipated allows us to study the pure effect of technology disentangled from the effect of preparing for an expected productivity increase. Also, even if a productivity increase is anticipated by the fishing industry it may still catch regulating authorities off guard. The present paper may thus inform regulators how to respond to such an event. It should also be noted that even if the interpretation of the change in the parameter in the present paper is as a productivity increase, other interpretations are also possible. E.g. an unanticipated permanent decrease in the price of an important normal input has the same effect on output as a productivity shock. See Bertoletti and Rampa (2013) for an analysis of what constitutes normal and inferior goods.

\section{The Canonical Schooling Fisheries Model}

The following is the basic version of the fisheries model applied to a schooling fishery (Clark 1990, p 97). In theoretical models of pure schooling fisheries harvest costs are not dependent on stock size (Neher 1990, p 177). We assume that the net instantaneous benefits from harvesting is given by a continuous and strictly concave function of the harvest rate, $h$, and given by $D(h)-C(h) \alpha^{-1}$. Here $D(h)$ is a benefit function, typically price multiplied by harvest rate, $C(h) \alpha^{-1}$ is a cost function and $\alpha$ is a parameter with higher $\alpha$ indicating better technology. It is assumed that $D(0)=C(0)=0 . D^{\prime}(h)$ and $C^{\prime}(h)$ are both assumed 
positive. $D^{\prime}(h)-C^{\prime \prime}(h) \alpha^{-1}$ is assumed negative and $C^{\prime \prime}(h)$ is assumed positive. We also make the important assumption that $0<D^{\prime}(0)-C^{\prime}(0) \alpha^{-1}<\infty$. This assumption implies that we are not considering a subsistence fishery, but rather a commercial fishery or a fishery where capital and labour have alternative uses.

The fish stock is assumed to be governed by

$$
\dot{x}=G(x)-h, x(0) \text { given }
$$

The natural growth function $G(x)$ is taken to be strictly concave, differentiable and satisfy $G(0)=G(K)=0$ for some $K>0$ and positive for all $x \in(0, K)$. The specification of $G(x)$ is in line with standard biological growth functions such as the logistic, which is used in phase portraits below, but the formal results do not require a parametric growth function. We assume there exists $x \in(0, K)$ such that the derivative of the growth function equals the discount rate, $G^{\prime}(x)=\rho$, which is reasonable for many commercially interesting fish species. The assumptions lead to the following optimization problem:

$$
\begin{aligned}
& V(x(0), \alpha)=\max _{h(t) \geq 0} \int_{0}^{\infty}\left(D(h)-C(h) \alpha^{-1}\right) e^{-\rho t} d t \\
& \text { subject to } \dot{x}=G(x)-h \text { and } x(0)>0 \text { given }
\end{aligned}
$$

The current value Hamiltonian for this problem is:

$$
H=D(h)-C(h) \alpha^{-1}+\mu(G(x)-h)
$$

The problem in (2) may be solved by using 4 sufficiency conditions for optimality found in e.g. Theorem 9.11.1 in Sydsæter et al. (2008). The first condition require that $h$, maximize the Hamiltonian at all $t$ which implies:

$$
\frac{\partial H}{\partial h}=D^{\prime}(h)-C^{\prime}(h) \alpha^{-1}-\mu \leq 0(=0 \text { if } h>0)
$$

The second condition requires that the co-state variable satisfy:

$$
\dot{\mu}=\left(\rho-G^{\prime}(x)\right) \mu
$$

The third condition is that $H$ is a concave function over $(h, x)$ which is straightforward to confirm. The fourth condition requires that we establish that:

$$
\lim _{t \rightarrow \infty} \mu(t) e^{-r t}(\tilde{x}(t)-x(t)) \geq 0
$$

This must hold for all admissible functions $\tilde{x}(t)$ that satisfies (1). This condition can only be checked after an optimal solution has been found. However, from the phase diagram in Fig. 1 it is clear that $\mu(t)<\infty$ as long as $x(0)>0$. We further have that and $0 \leq \tilde{x}(t) \leq K$ for all possible $\tilde{x}(t)$ satisfying the differential equation, so the left hand side of (6) computes to zero for all possible $\tilde{x}(t)$.

Equation (4) defines $h$ as a function of $\mu$ and $\alpha$. This function is denoted ${ }^{1}$ :

\footnotetext{
${ }^{1}$ It is also implicitly assumed that the value of $h$ that solves $D^{\prime}(h)-C^{\prime}(h) \alpha^{-1}=0$ is larger than $h_{s s}$. Without this assumption there is no need to regulate the fishery as the optimal shadow price is zero.
} 


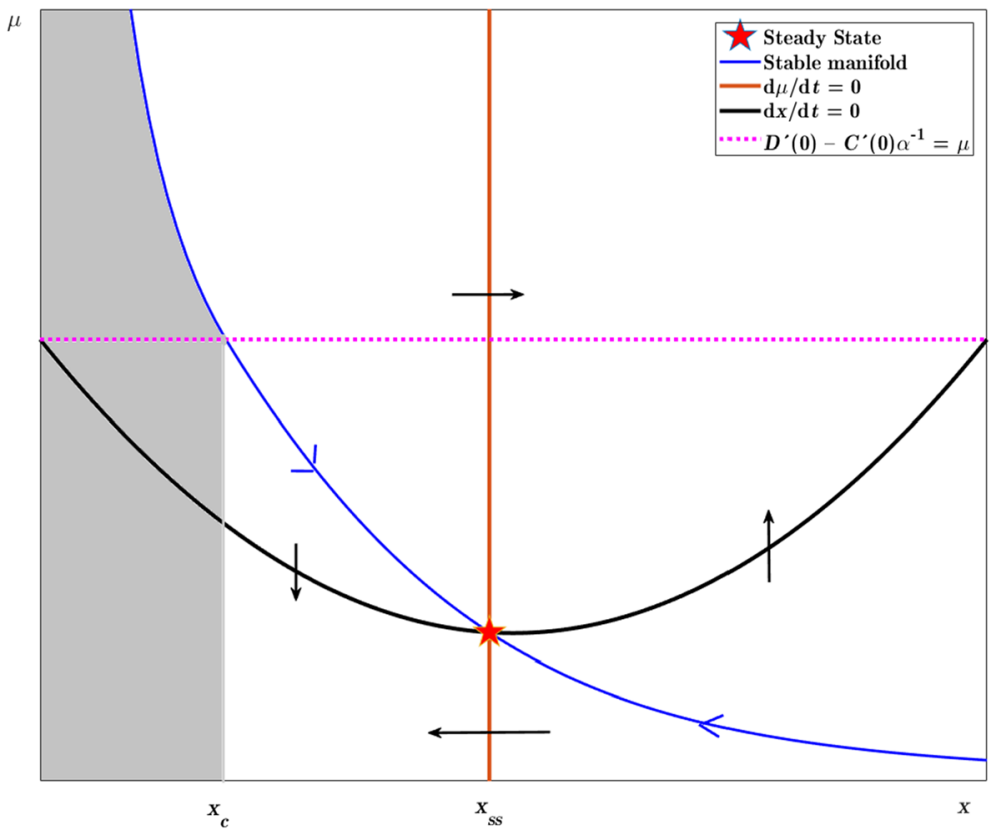

Fig. 1 A phase portrait for an optimally managed fishery. The figure is computer generated and uses a model where $D(h)-C(h) \alpha^{-1}=p h-\frac{c}{2 \alpha} h^{2}$ and $G(x)=r x(1-x / K)$. The intersection between the isocline where $\mathrm{d} x / \mathrm{d} t=0$ and the isocline where $\mathrm{d} \mu / \mathrm{d} t=0$ defines the steady state which is a saddle point. Convergence towards the steady state is along the stable manifold that shows combinations of $x$ and $\mu$ that are compatible with optimality. There is a critical value of $x, x_{c}$, such that harvesting is zero for all $x \leq x_{c} \cdot x_{c}$ is determined by the intersection of the stable manifold and the line $\mu=D^{\prime}(0)-C^{\prime}(0) \alpha^{-1}$. The stable manifold, $\mu(x, \alpha)$, is the derivative of the value function and the value of the fishery is zero when the stock is zero. This implies that the value function, $V(x)$, is given by the integral under the stable manifold. Thus $V\left(x_{c}\right)$, indicated by the grey area, is the value of the fishery at the stock level where it is optimal for the fishery to commence. Parameter values are given by $p=5, c=3, r=1, K=10, \rho=0.05$ and $\alpha=2$

$$
h=\phi(\mu, \alpha)
$$

Note that (4) and Assumption 1 implies that $D^{\prime}(0)-C^{\prime}(0) \alpha^{-1}<\mu \Rightarrow \phi(\mu, \alpha)=0$. For all $x$ where (4) holds with equality it also holds that:

$$
\begin{gathered}
\frac{\partial h}{\partial \mu}=\phi_{\mu}^{\prime}=\frac{1}{D^{\prime \prime}(h)-C^{\prime \prime}(h) \alpha^{-1}}<0 \\
\frac{\partial h}{\partial \alpha}=\phi_{\alpha}^{\prime}=-\frac{C^{\prime}(h) \alpha^{-2}}{D^{\prime \prime}(h)-C^{\prime \prime}(h) \alpha^{-1}}>0 \text { for } h>0 \\
\left(\frac{\partial \mu}{\partial \alpha}\right)_{h \text { is constant }}=-\frac{\phi_{\alpha}^{\prime}}{\phi_{\mu}^{\prime}}=C^{\prime}(h) \alpha^{-2}>0 \text { for } h>0
\end{gathered}
$$


The inequality in (9) may lead us to infer that higher productivity implies larger harvest rates. As we shall see, the inequality (10) and how it affects the shape of the isocline for $\dot{x}=0$ is part of the reason why this is wrong.

The model assumptions ensure that there exists a steady state $\left(h_{s s}, x_{s s}, \mu_{s s}\right)=\lim _{t \rightarrow \infty}(h(t)$, $x(t), \mu(t))>0$ which if $x(0)>0$ will be the long run equilibrium for an optimally managed fishery (Clark 1973; Cropper 1979). Convergence of $(h(t), x(t), \mu(t))$ to the steady state $\left(h_{s s}, x_{s s}, \mu_{s s}\right)$ will be along a stable saddle path towards a steady state characterized by $\dot{x}=G(x)-\phi(\mu, \alpha)=0$ and $\dot{\mu}=\left(\rho-G^{\prime}(x)\right) \mu=0$. This stable path can be found in $(x, \mu)$ space by solving the following differential equation (Conrad and Clark 1987, Ch. 1.6.6; Judd 1998, Ch. 10.7):

$$
\frac{\dot{\mu}}{\dot{x}}=\frac{\mathrm{d} \mu}{\mathrm{d} x}=\frac{\left(\rho-G^{\prime}(x)\right) \mu}{G(x)-\phi(\mu, \alpha)}, \mu\left(x_{s s}, \alpha\right)=\mu_{s s}
$$

The solution to (11) defines $\mu$ as a function of $x$ along an optimal path. We denote the stable saddle path as $\mu(x, \alpha)$. It is worthwhile to note that the stable saddle path $\mu(x, \alpha)$ is in fact the derivative of the value function, $V(x, \alpha)$. As $V(0, \alpha)=0$ in this model, we can illustrate the value function in a phase portrait as the integral under the stable manifold. A phase portrait of the optimal solution is shown in Fig. 1.

Inserting $\mu(x, \alpha)$ into (7) gives us optimal $h$ as a feedback control:

$$
h=\phi(\mu(x, \alpha), \alpha)=\Phi(x, \alpha)
$$

The expression in (12) is a feedback control and gives us optimal harvest rates as a function of the stock and the productivity parameter and is usually derived in a dynamic programming framework. However, by using optimal control with its explicit focus on the shadow price we gain some additional structure to the problem that enables us to construct analytical results not directly accessible with dynamic programming where interior solutions are typically assumed. ${ }^{2}$

One such result, crucial in the analysis below, may be found in Nævdal and Skonhoft (2018) who prove that if $0<D^{\prime}(0)-C^{\prime}(0) \alpha^{-1}<\infty$ there exists a critical stock level $x_{c}>0$ such that $h=\Phi(x, \alpha)=0$ for all $x \leq x_{c}$. In other words; as long as the instantaneous marginal utility of harvesting is finite at $h=0$, it is optimal for a well managed fishery to temporarily close down if fish stocks are sufficiently low.

\section{The Effect of Increased Productivity}

Here we demonstrate that for a given stock size increased productivity reduces the optimal harvest rate if $h$ is positive and the stock is below the steady state level, i.e. $\phi(x, \alpha)$ is a decreasing function of $\alpha$ for all $x \in\left(x_{c}, x_{s s}\right)$. This result is built on 4 propositions that are given below. However a fairly rigorous explanation is given in Fig. 2.

In order to lay the ground for the propositions that follows, note that an increase in $\alpha$ will shift the $\dot{x}=0$-isocline in Fig. 1 upwards for all $x$ except $x=0$ and $x=K$. For

\footnotetext{
2 Alternatively we could use the optimal control conditions in (4), (5) and the differential equation for $x$ to construct a differential equation $\dot{h}=v(h, x)$, see Eq. (13) below. This equation would however only be valid when (4) has an interior solution and is not very helpful here as much of the present analysis deals with the case where $h=0$ is the solution to (4) along the optimal path.
} 


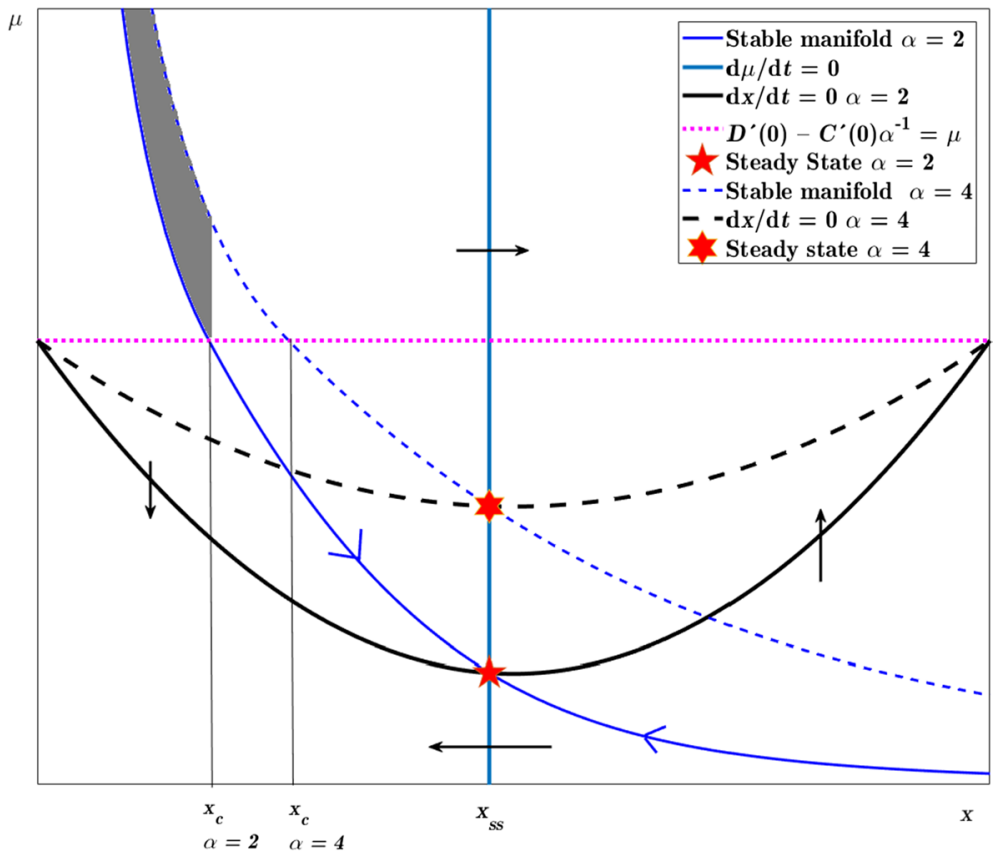

Fig. 2 The effect of technological change in a standard phase portrait. The model and parameters are as in Fig. 1. The technological change consists of $\alpha$ increasing from 2 to 4 . The isocline for $\mathrm{d} \mu / \mathrm{d} t=0$ and the line $\mu=D^{\prime}(0)-C^{\prime}(0) \alpha^{-1}$ are not affected. The isocline for $\mathrm{d} x / \mathrm{d} t=0$ shifts upward as indicated by the shift from the solid black line to the dashed black line. This changes the steady state from the point indicated by to the point indicated by Thus the distance between the steady state and the line $\mu=D^{\prime}(0)-C^{\prime}(0) \alpha^{-1}$ decreases and the steady state value of $\mu$ increases. It follows from Proposition 1 that at the stable manifolds for high and low productivity cannot intersect for any $x\left(0, x_{s s}\right)$. These factors taken together imply Proposition 2 ; the stable manifold with high productivity lies above the stable manifold for low productivity for all $x\left(0, x_{s s}\right)$. Proposition 3 follows; $x_{c}$ increases in response to a productivity increase. Propositions 1,2 and 3 can then be used to prove Proposition 4. The economic explanation for Propositions 3, 4 and 5 is that higher productivity increases the value of current stock growth as the value of future harvests increase. The shaded area indicates the increase in the objective function resulting from the productivity improvement if $x(0)=x_{c}$ for $\alpha$ low

any given $x$ in $(0, K)$, then along the $\dot{x}=0$-isocline we have that $h=\phi(\mu, \alpha)=G(x)$ is a positive constant. From (10) it follows that if $\alpha$ increases, then $\mu$ must increase if $h$ is to remain constant except at $x=0$ and $x=K$ as $C^{\prime}(0)=0$ implies that $\mu$ is unaffected by the change in $\alpha$ at these points.

\section{Proposition}

1 Define

$Q(\alpha)=\left\{(x, \mu) \mid 0<x<x_{s s}, \dot{x}=G(x)-\phi(\mu, \alpha)>0,0<\mu<D^{\prime}(0)-C^{\prime}(0) \alpha^{-1}\right\}$. Let $\alpha^{\text {high }}>\alpha^{\text {low }}$. As the $\dot{x}=0$ isocline shifts upwards as $\alpha$ increases it follows that $Q\left(\alpha^{\text {high }}\right)$ is a subset of $Q\left(\alpha^{\text {low }}\right)$. Then for any point $(x, \mu) \in Q\left(\alpha^{\text {high }}\right)$ it holds that $(\mathrm{d} \mu / \mathrm{d} x)_{\alpha^{h i g h}}<(\mathrm{d} \mu / \mathrm{d} x)_{\alpha^{l o w}}<0$.

Proof The slope of any solution through an arbitrary point in $Q\left(\alpha^{\text {high }}\right)$ is given by: 


$$
\frac{\dot{\mu}}{\dot{x}}=\frac{\mathrm{d} \mu}{\mathrm{d} x}=\frac{\left(\rho-G^{\prime}(x)\right) \mu}{G(x)-\phi(\mu, \alpha)}
$$

This is true for all $\alpha \in(0, \infty)$. From (10) we have that $\phi(\mu, \alpha)$ is an increasing function of $\alpha$ when $(x, \mu) \in Q\left(\alpha^{\text {high }}\right)$ and the conclusion follows.

Proposition 1 defines a set $Q(\alpha)$. This set is significant in the present context because the stable manifold, $\mu(x, \alpha)$, is contained in $Q(\alpha)$ for all $x \in\left(0, x_{s s}\right)$. What this proposition does is to take an arbitrary point in $Q\left(\alpha^{\text {high }}\right)$, calculate the slope of any solution that passes through that point and show that the slope is steeper when $\alpha$ is higher. Proposition 1 can be used to prove the following proposition:

Proposition 2 (a) $\mu(x, \alpha)$ is an increasing function of $\alpha$ for all $x \in\left(0, x_{s s}\right]$.

Proof As a productivity increase shifts the $\dot{x}=0$ isocline upwards while the $\dot{\mu}=0$ isocline is unchanged, it follows that $\mu\left(x_{s s}, \alpha^{\text {high }}\right)>\mu\left(x_{s s}, \alpha^{\text {low }}\right)$. If the proposition is false, then there must exist an $x^{\prime} \in\left(0, x_{s s}\right)$ such that $\mu\left(x^{\prime}, \quad \alpha^{h i g h}\right)=\mu\left(x^{\prime}, \quad \alpha^{l o w}\right)$ and $(\mathrm{d} \mu / \mathrm{d} x)_{\alpha=\alpha^{\text {high }}}<(\mathrm{d} \mu / \mathrm{d} x)_{\alpha=\alpha^{\text {low }}}$. From Proposition 1 it follows that $(\mathrm{d} \mu / \mathrm{d} x)_{\substack{\alpha=\alpha^{\prime} \\ x=x^{\prime}}}^{\substack{\text { high } \\ x=x^{\prime}}}>(\mathrm{d} \mu / \mathrm{d} x)_{\substack{\alpha=\alpha^{\prime} \\ x=x^{\prime}}}^{\substack{x=x^{\prime} \\ x}}$ which is a contradiction.

Given the economic interpretation of the stable manifold as the derivative of the value function, this also makes intuitive sense; better technology increases the marginal value of the stock given the stock size.

The critical stock level $x_{c}$ at and below which optimal $h$ is zero will also be affected by a productivity increase. We can prove that $x_{c}$ is in fact an increasing function of $\alpha$.

Proposition 3 The critical $x_{c}$ where $h=0$ for all $x<x_{c}$ is an increasing function of $\alpha$.

Proof Define the relationship between $x_{c}$ and $\alpha$ by $x_{c}=x_{c}(\alpha)$. From Proposition 2 we have that $D^{\prime}(0)-C^{\prime}(0) \alpha^{-1}=\mu\left(x_{c}\left(\alpha^{l o w}\right), \alpha^{l o w}\right)<\mu\left(x_{c}\left(\alpha^{l o w}\right), \alpha^{\text {high }}\right)$. It follows that the value of $x_{c}\left(\alpha^{\text {high }}\right)$ that solves $D^{\prime}(0)-C^{\prime}(0) \alpha^{-1}=\mu\left(x_{c}\left(\alpha^{\text {high }}\right), \alpha^{\text {high }}\right)$ is larger than $x_{c}\left(\alpha^{\text {low }}\right)$.

We can now prove the main result in this section.

Proposition 4 Let $\alpha^{\text {low }}<\alpha^{\text {high }}$. Then the feedback control $\phi(x, \alpha)$ has the property that for all $x<x_{s s}$ it holds that $\Phi\left(x, \alpha^{\text {high }}\right) \leq \Phi\left(x, \alpha^{\text {low }}\right)$.

Proof Denote $x_{c}\left(\alpha^{i}\right)$ as $x_{c}^{i}$ for $i=\{$ high, low $\}$. A consequence of the existence of $x_{c}$ and Proposition 3 is that there exists some $x_{c}^{\text {low }}$ such that $\Phi\left(x_{c}^{\text {low }}, \alpha^{\text {low }}\right)=\Phi\left(x_{c}^{\text {high }}, \alpha^{\text {high }}\right)=0$ for all $x \in\left[0, x_{c}^{\text {low }}\right]$ and that there is an interval $\left(x_{c}^{\text {low }}, x_{c}^{\text {high }}\right]$ where $\Phi\left(x, \alpha^{\text {low }}\right)>\Phi\left(x, \alpha^{\text {high }}\right)=0$. It follows from (5) that $x_{s s}$ is determined and independent of $\alpha$. It follows from $\dot{x}=G\left(x_{s s}\right)-h=0$ in steady state that $h_{s s}$ is also independent of $\alpha$, thus $\Phi\left(x_{s s}, \alpha^{h i g h}\right)=\Phi\left(x_{s s}, \alpha^{\text {low }}\right)$. We must now account for the open interval $\left(x_{c}^{\text {high }}, x_{s s}\right)$. If $\Phi\left(x, \alpha^{\text {low }}\right)<\Phi\left(x, \alpha^{h i g h}\right)$ for some subinterval of $\left(x_{c}^{\text {high }}, x_{s s}\right)$ there must exist a pair $(\tilde{h}, \tilde{x})$ where $\tilde{x} \in\left(x_{c}^{\text {high }}, x_{s s}\right)$ such that $\tilde{h}=\Phi\left(\tilde{x}, \alpha^{\text {low }}\right)=\Phi\left(\tilde{x}, \alpha^{\text {high }}\right)$ and $\Phi_{x}^{\prime}\left(\tilde{x}, \alpha^{\text {low }}\right)<\Phi_{x}^{\prime}\left(\tilde{x}, \alpha^{\text {high }}\right)$. We can calculate $\Phi_{x}^{\prime}(x, \alpha)$ in a point $(\tilde{h}, \tilde{x})$ and when we insert from (8) and (11) we get that: 


$$
\Phi_{x}^{\prime}(\tilde{x}, \alpha)=\left(\phi_{\mu}^{\prime} \frac{\mathrm{d} \mu}{\mathrm{d} x}\right)_{(h, x)=(\tilde{h}, \tilde{x})}=\underbrace{\frac{1}{D^{\prime \prime}(\tilde{h})-C^{\prime \prime}(\tilde{h}) \alpha^{-1}}}_{<0} \underbrace{\frac{\left(\rho-G^{\prime}(\tilde{x})\right) \mu(\tilde{x}, \alpha)}{G(\tilde{x})-\tilde{h}(\mu(\tilde{x}, \alpha), \alpha)}}_{<0}>0
$$

We now compare $\Phi_{x}^{\prime}\left(\tilde{x}, \alpha^{l o w}\right)$ and $\Phi_{x}^{\prime}\left(\tilde{x}, \alpha^{h i g h}\right)$ to see under what condition the same harvest rate at the same stock level yields $\Phi_{x}^{\prime}\left(\tilde{x}, \alpha^{l o w}\right)<\Phi_{x}^{\prime}\left(\tilde{x}, \alpha^{h i g h}\right)$. We calculate that

$$
\begin{aligned}
& \Phi_{x}^{\prime}\left(\tilde{x}, \alpha^{\text {low }}\right)<\Phi_{x}^{\prime}\left(\tilde{x}, \alpha^{h i g h}\right) \\
& \mathbb{1} \\
& \frac{1}{D^{\prime \prime}(\tilde{h})-C^{\prime \prime}(\tilde{h}) / \alpha^{\text {low }}} \frac{\left(\rho-G^{\prime}(\tilde{x})\right) \mu\left(\tilde{x}, \alpha_{\text {low }}\right)}{G(\tilde{x})-\tilde{h}}<\frac{1}{D^{\prime \prime}(\tilde{h})-C^{\prime \prime}(\tilde{h}) / \alpha^{h i g h}} \frac{\left(\rho-G^{\prime}(\tilde{x})\right) \mu\left(\tilde{x}, \alpha^{h i g h}\right)}{G(\tilde{x})-\tilde{h}} \\
& \mathbb{1} \\
& \frac{\mu\left(\tilde{x}, \alpha_{\text {low }}\right)}{D^{\prime \prime}(\tilde{h})-C^{\prime \prime}(\tilde{h}) / \alpha^{\text {low }}}>\frac{\mu\left(\tilde{x}, \alpha^{\text {high }}\right)}{D^{\prime \prime}(\tilde{h})-C^{\prime \prime}(\tilde{h}) / \alpha^{\text {high }}} \\
& \frac{\mu\left(\tilde{x}, \alpha_{\text {low }}\right)}{D^{\prime \prime}(\tilde{h})-C^{\prime \prime}(\tilde{h}) / \alpha^{\text {low }}}<\frac{\mu\left(\tilde{x}, \alpha^{h i g h}\right)}{D^{\prime \prime}(\tilde{h})-C^{\prime \prime}(\tilde{h}) / \alpha^{\text {high }}}
\end{aligned}
$$

But the last inequality holds for all $(\tilde{h}, \tilde{x})$ including $\left(h_{s s}, x_{s s}\right)$ as $\mu(x, \alpha)$ is increasing in $\alpha$ and the denominator is decreasing in $\alpha{ }^{3}$ This implies that there is at most one point $(\tilde{h}, \tilde{x})$ where $\Phi\left(x, \alpha^{l o w}\right)=\Phi\left(x, \alpha^{h i g h}\right)$ and we already know that $\Phi\left(x_{s s}, \alpha^{l o w}\right)=\Phi\left(x_{s s}, \alpha^{h i g h}\right)$. It follows that there are no values of $x$ less than $x_{s s}$ such that the corresponding harvest rate is larger with larger productivity.

Thus we have that in a schooling fishery a unanticipated technological shock will lead to lower catch for each given stock size, in turn ensuring a faster convergence to the steady state. To get some intuition about this result note that the canonical fisheries model is very similar to the well known Ramsey-Cass-Koopmans (RCK) model of economic growth. The only difference is that marginal productivity at zero use of input is assumed infinity in the RCK model, whereas the corresponding expression in the fisheries model, $G^{\prime}(0)$, is assumed finite. In the analysis of the RCK model the instantaneous elasticity of substitution, also known by its reciprocal, the intertemporal elasticity of substitution is an important explanatory factor. Roughly speaking, the higher the instantaneous elasticity of substitution, the more willing is the consumer to reduce consumption in the present in order to increase consumption in the future, see Blanchard and Fischer (1989, pp 39-43) for a discussion of the instantaneous elasticity of substitution and how it affects intertemporal tradeoffs in the RCK-model. If we can show that a productivity increase has the same effect as an increase in the instantaneous elasticity of substitution we have a way of understanding and generalising the above results to a more general cost function $c(h, \alpha)$. It is assumed that $c(0, \alpha)=0$ and that $c(h, \alpha)$ is increasing and concave with respect to $h$. It is also assumed that $c(h, \alpha)$ and $c_{\alpha}^{\prime}(h, \alpha)$ are decreasing with respect to $\alpha$ except when $h=0$.

\footnotetext{
${ }^{3}$ It should be noted that in steady state the expression $\mu_{s s}\left(\rho-G^{\prime}\left(x_{s s}\right)\right) /\left(G\left(x_{s s}\right)-h_{s s}\right)$ is not defined. However, the numerical value of this expression does exist and may be found using L'Hôpital's rule. For our purposes it is sufficient to note that the numerical value exists and is negative.
} 
In the present model we can calculate the equivalent to the instantaneous elasticity of substitution for the integrand in (2). This expression is given by:

$$
\sigma(h, \alpha)=-\frac{D^{\prime}(h)-c_{h}^{\prime}(h, \alpha)}{h\left(D^{\prime \prime}(h)-c_{h h}^{\prime \prime}(h, \alpha)\right)}>0 \text { for all } h>0
$$

By using (4), (5) and the differential equation for $x$ one can derive the following differential equation for $h$ as a function of $x$ which holds when (4) has an interior solution.

$$
\frac{\dot{h}}{h}=\sigma(h, \alpha)\left(\rho-G^{\prime}(x)\right)
$$

In macroeconomics this equation is known as the Keynes-Ramsey rule. From (15) it is clear that for any given $x<x_{s s}$, the higher the numerical value of $\sigma(h, \alpha)$ the faster is the rate of convergence towards the steady state value of $h_{s s}$, and therefore also towards $x_{s s}$. Differentiating $\sigma(h, \alpha)$ with respect to $\alpha$ yields:

$$
\frac{\partial \sigma}{\partial \alpha}=\frac{\overbrace{c_{h \alpha}^{\prime \prime}(h, \alpha)}^{<0} \overbrace{(D^{\prime \prime}(h)-\underbrace{\left.c_{h h}^{\prime \prime}(h, \alpha)\right)}_{>0}}^{<0}+\overbrace{\left.\left(c_{h}^{\prime}(h, \alpha)-D^{\prime}(h)\right)\right)}^{<0} \overbrace{c_{h h \alpha}^{\prime \prime \prime}(h, \alpha)}^{\text {sign }=?}}{\underbrace{h\left(D^{\prime \prime}(h)-c_{h h}^{\prime \prime}(h, \alpha)\right)^{2}}_{>0}}
$$

With exception of $c_{h h \alpha}^{\prime \prime \prime}$, the sign of all terms in this expression follows from assumptions, first order conditions and second order conditions. It follows that $c_{h h \alpha}^{\prime \prime \prime}<0$ is a sufficient, but not a necessary condition for $\sigma$ to be an increasing function of $\alpha$ and therefore for Propositions 3 and 4 to hold also with this more general cost function. Thus improved productivity has the same qualitative effect along the segment of the optimal path where $h>0$ as an increase in the instantaneous elasticity of substitution has in the RCK model. Intuitively, this condition implies that the second derivative of costs with respect to $h$ decreases in response to improved technology. As the steady state harvest and stocks level are fixed, this implies that net instantaneous utility increases more for high harvest levels close to the steady state than harvest levels further below steady state, which makes it optimal to sacrifice some instantaneous net utility in the near future in order to speed up the rate of convergence towards steady state. Remarkably as long as $c_{h h \alpha}^{\prime \prime \prime}<0$, this effect does not depend on the discount rate. It should be pointed out that although the canonical model of a schooling fishery and the RCK model are mathematically almost equivalent, they are of course models of very different things. Importantly, the RCK model has utility as the unit and $\rho$ is the rate of time preference. In the fisheries model as long as we do not examine a subsistence fishery, but a commercial fishery the unit is money and the discount rate is the interest rate.

\section{Stock Dependent Costs}

We now look at the case where the harvest costs depend on stock size, reflecting that for many fish-species it is easier to catch a unit of fish if the stock is abundant and if the stock of the fish goes to zero, then the marginal cost of harvesting a unit of fish goes to infinity. The canonical model is now: 


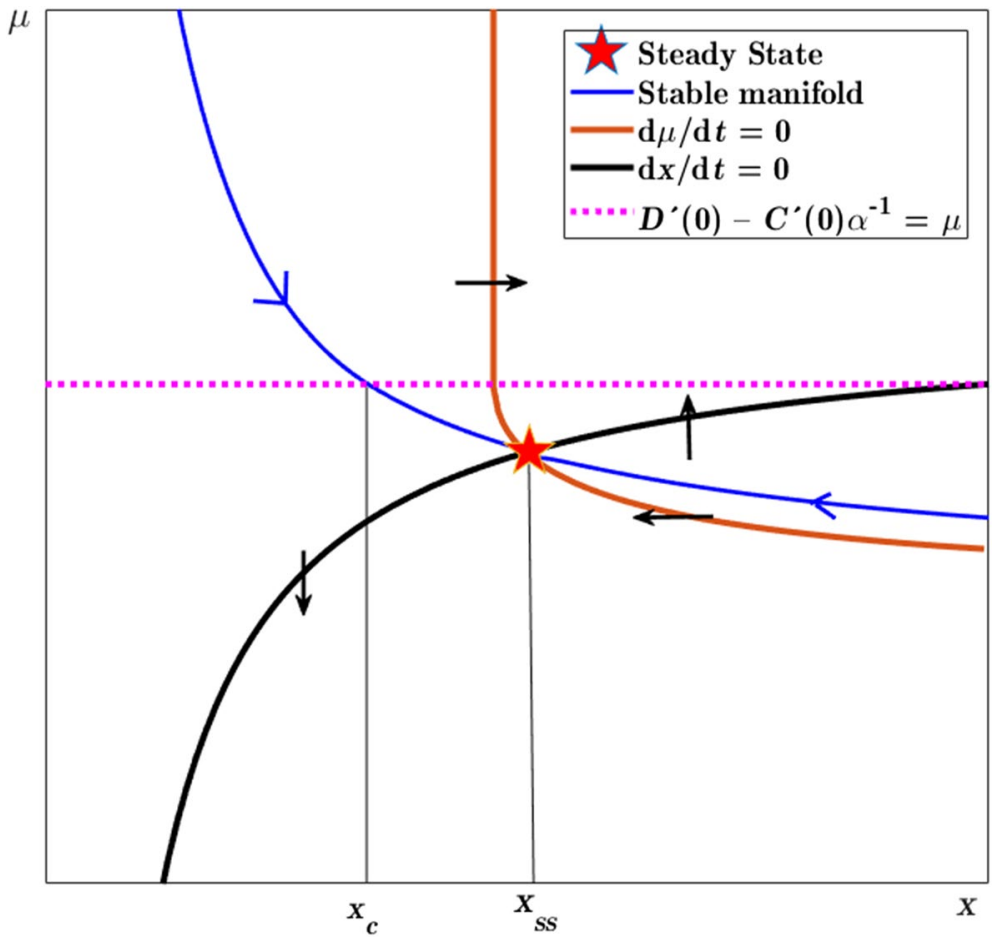

Fig. 3 Phase portrait when costs are stock dependent. The figure is computer generated and uses a model where $D(h)-C(h, x) \alpha^{-1}=p h-1 / 2 c(h / x)^{2} \alpha^{-1}$ and $G(x)=r x(1-x / K)$. We then have that $D^{\prime}(0)-C_{h}^{\prime}(0, x, \alpha)=p$. The major difference between this portrait and the portrait when harvesting costs are independent of stock size is the shape of the isoclines. The qualitative effect on the general shape of the stable manifold is minor. Note that for $x \leq x_{c}$ where $x_{c}$ is defined by where the stable manifold intersects the line where $\mu=p$, optimal harvest becomes zero. The value of $x$ that solves $\rho=G^{\prime}(x)$ is a lower bound for the steady state stock regardless of how technology affects the cost function. Thus, there is a limit to how much stock dependent costs can affect the stable manifold at values of $x$ below this lower bound as optimal $h$ will become zero very quickly below it. Parameter values are given by $p=5, c=3, r=1, K=10, \rho=0.05$ and $\alpha=3 / 4$

$$
\begin{aligned}
& V(x(0), \alpha)=\max _{h \geq 0} \int_{0}^{\infty}(D(h)-C(h, x, \alpha)) e^{-\rho t} d t \\
& \text { subject to } \dot{x}=G(x)-h \text { and } x(0) \text { given. }
\end{aligned}
$$

It is assumed that $C_{h}^{\prime}(h, x, \alpha)>0, C_{x}^{\prime}(h, x, \alpha)<0, C_{h \alpha}^{\prime \prime}(h, x, \alpha)<0$ for all $h$ and $x>0$, $C_{h \alpha}^{\prime \prime}(0, x, \alpha)=0$ and that $C_{h h}^{\prime \prime}(h, x, \alpha)>0$. This is consistent with, but not restricted to, cost functions of the form ${ }^{4}$

\footnotetext{
${ }^{4}$ The cost function in (16) may be derived from a generalized Schaefer harvest function $h=q E^{\gamma_{1}} x^{\gamma_{2}}$ where $E$ is effort and $q, \gamma_{1}$ and $\gamma_{2}$ are parameters. If the cost of effort is given by $k E$, then (16) follows from letting $\alpha=q^{\gamma_{1}^{-1}}, a=\gamma_{1}^{-1}$ and $b=\gamma_{2} \gamma_{1}^{-1}$, see Morey (1986) and Clark (1990).
} 


$$
C(h, x, \alpha)=k \frac{h^{a}}{\alpha x^{b}} \text { where } a>1 \text { and } b>0
$$

Thus the technology is such that an increase in $\alpha$ lowers the marginal cost of harvesting. It follows from $C(0, x, \alpha)=0$ and $C_{h \alpha}^{\prime \prime}(h, x, \alpha)<0$ that an increase in $\alpha$ also reduces costs for all positive $h$ and $x$.

This model or models very similar to this one has been analysed in a large number of papers and is close to the analysis of the pure schooling fishery above. A typical phase diagram is shown in Fig. 3.

We keep the notation from the previous analysis so $\phi(\mu, \alpha)$ is the harvest rate that maximises the Hamiltonian, $\mu(x, \alpha)$ is the stable manifold/derivative of the value function, $\phi(x$, $\alpha$ ) is optimal harvest rate as a feedback control and $x_{c}^{j}$ is the critical stock level below which one should not harvest when the productivity is of type $j$.

With stock dependent costs there is also a critical value $x_{c}$ determined by the lowest stock of $x$ such that the first order condition for the maximised Hamiltonian is that its derivative with respect to $h$ is zero 5 :

$$
\frac{\partial H}{\partial h}=D^{\prime}(0)-C_{h}^{\prime}\left(0, x_{c}, \alpha\right)-\mu=0
$$

We can use this to find conditions that ensure that $x_{c}$ is a increasing function of $\alpha$.

Proposition $5 C_{h x}^{\prime \prime}(0, x, \alpha) \leq 0$ is a sufficient, but not necessary condition for $x_{c}$ being an increasing function of $\alpha$.

Proof Inserting $\mu(x, \alpha)$ into (19) and implicitly differentiating gives the result:

$$
\frac{\partial x_{c}}{\partial \alpha}=\frac{\overbrace{\frac{\partial \mu\left(x_{c}, \alpha\right)}{\partial \alpha}}^{>0}}{(\underbrace{C_{h x}^{\prime \prime}\left(0, x_{c}, \alpha\right)}_{\operatorname{sign}=?}+\underbrace{\frac{\partial \mu\left(x_{c}, \alpha\right)}{\partial x}}_{<0})}
$$

If $C_{h x}^{\prime \prime}(0, x, \alpha) \leq 0$ then the expression in (20) is positive.

Assuming that $C_{h x}^{\prime \prime}(0, x, \alpha) \leq 0$ is in line with standard parameterisations of cost functions used in fisheries. It is also reasonable to expect that the higher the stock, the lower is the marginal cost with respect to $h$. However, one can not rule out that there are exceptions to this rule. One can e.g. envision changes in technology that improves productivity for some values of $h$ and $x$ and reduces it for others. If that is the case a more detailed numerical investigation is warranted to establish the sign of $\partial x_{c} / \partial \alpha$.

\footnotetext{
${ }^{5}$ The proof for this statement is much simpler than with the model without state dependent harvest cost given in Nævdal and Skonhoft (2018). Here it suffices to note that as long as marginal cost goes to infinity as $x$ goes to zero and marginal revenue $D(0)$ is a positive finite number, such a critical $x_{c}$ will exist (Leung and Wang 1976).
} 


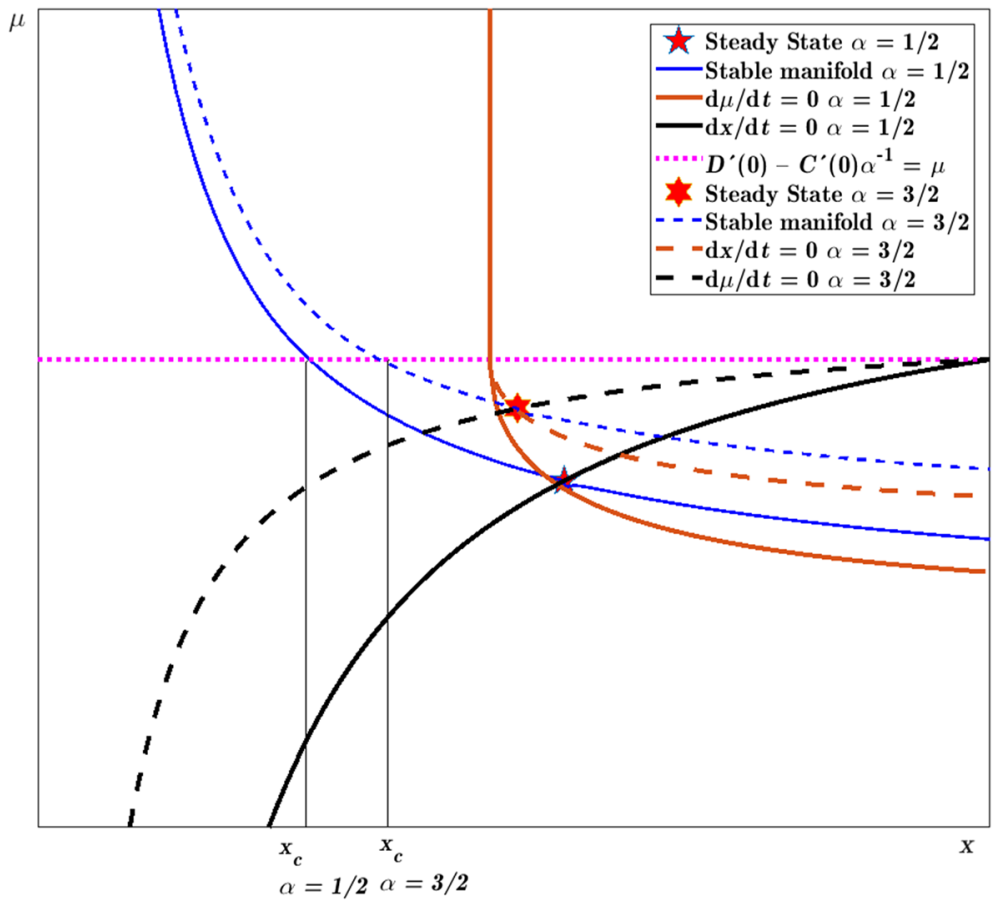

Fig. 4 The effect of a productivity increase with stock dependent costs. The figure is computer generated with the same parameterization as Fig. 3 except that $\alpha$ is $1 / 2$ when productivity is low and jumps to $\alpha=3 / 2$. In line with Propositions 5 and 6, $x_{c}$ increase as a function of $\alpha$ and reduce harvests for stock levels close to $x_{c}$. At the same time increased productivity decreases the steady state $x_{s s}$, so, in line with results in Caputo (1989), at least for a period it holds that higher productivity increases harvest rates if the stock is close to the steady state. At the limit, as $\alpha \rightarrow \infty, x_{c}$ and $x_{s s}$ will converge to the same point determined by $\rho=G^{\prime}(x)$

\subsection{The Effect of Improved Productivity on Optimal Harvest Rate when $x>x_{c}$}

Clark and Munro (1975) demonstrate in a model that is linear in the harvest rate that an increase in productivity will lead to lowered steady state stocks. Caputo (1989) demonstrates in a fairly general non-linear model that the long-run effect of a productivity increase is also a lower stock level. Caputo (1989) also show and that a productivity increase may be commensurate with both a higher and a lower long term harvest rate $h_{s s^{*}}$. The initial response will however be an increase in the harvest rate. The results in Caputo (1989) are based on the response to a productivity shock if the initial stock is in steady state. However, even if the steady state response to a positive productivity shock implies that the steady state stock decreases when $\alpha$ increases it is still the case that $\phi(x, \alpha)$ will be a decreasing function of $\alpha$ if $x$ sufficiently small as proven in Proposition 6.

Proposition 6 If Proposition 5 holds, an increase productivity from $\alpha^{\text {low }}$ to $\alpha^{\text {high }}$ implies that there is an non-empty interval $\left[x_{c}^{\text {low }}, \hat{x}\right)$ such that harvest levels for a given stock size is reduced, i.e. $\phi\left(x, \alpha^{\text {high }}\right)<\phi\left(x, \alpha^{\text {low }}\right)$ for all $x$ in $\left[x_{c}^{l o w}, \hat{x}\right)$. 


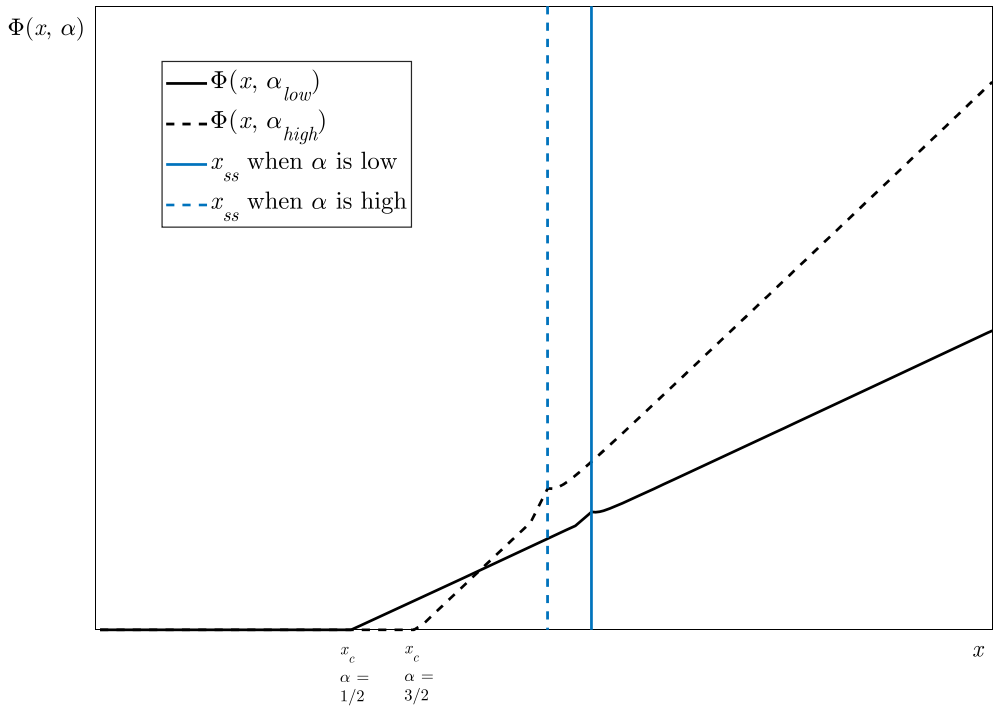

Fig. 5 The effect of a productivity increase on harvest as function of stock for stock levels above and below the steady state. Same parameter values as in Figs. 3 and 4. The solid lines indicate the situation before productivity increase. The dashed lines shows harvest and steady state after productivity increase. As the model satisfies the premise of Proposition 5, $x_{c}$ increases as a response to the productivity shock even if the optimal steady state decreases. As predicted by Proposition 6, there is an interval where the productivity increase leads to lower harvest levels even if harvest levels are increased for stock sizes just below steady state. Above the steady state stock level we have that increased productivity always leads to higher harvest levels for a given stock size

Proof It follows from the existence of $x_{c}>0$ for all $\alpha>0$ and Proposition 6 that there exists a non-empty intervals $\left[0, x_{c}^{l o w}\right]$ such that $\phi\left(x, \alpha^{\text {low }}\right)=\phi\left(x, \alpha^{\text {high }}\right)=0$ and another non-empty interval $\left[x_{c}^{\text {low }}, x_{c}^{\text {high }}\right]$ where $\phi\left(x, \alpha^{\text {low }}\right)>0$ and $\phi\left(x, \alpha^{\text {high }}\right)=0$. Note that $h=\phi(x, \alpha)$ is a continuous function of $x$ and that $\phi\left(x_{c}^{\text {high }}, \alpha^{\text {low }}\right)>\phi\left(x_{c}^{\text {high }}, \alpha^{\text {high }}\right)=0$. Due to continuity, $h$ cannot jump infinitely fast at $x_{c}^{\text {high }}$ and there is therefore an interval $\left(x_{c}^{\text {high }}, \hat{x}\right]$ where $\phi\left(x, \alpha^{l o w}\right)>\phi(x$, $\left.\alpha^{h i g h}\right)>0$.

This effect remains regardless of the size of productivity increase. An important thing to note from Fig. 3 is that there is a lower bound to how low the steady state stock will be reduced by improved technology. Proposition 6 shows that below this lower bound, there will be an interval $\left[x_{c}^{l o w}, \hat{x}\right)$ where the higher the productivity, the lower the harvest rate. The effect of increased productivity is summarized in Fig. 4.

Figure 4 shows that the results in the Clark and Munro (1975), Caputo (1989) and Squires and Vestergaard (2013) where increased productivity leads to lower steady state stocks are compatible with the results in the present paper. If increased productivity increases rents in steady state and the stock is low enough one should still decrease harvesting in order to increase stocks faster even if the desired steady state level has decreased. This is illustrated in Fig. 5.

Squires and Vestergaard (2013) also present results where improved technology leads to short term decreases in harvesting. However, their mechanism is that if the regulator knows there will be technology improvements in the future, then it will pay to reduce harvest rates 
until the new technology can be utilized. In the present paper the technology improvement is unanticipated, but the improvements are still best utilized by letting the fish stock grow faster even though instantaneous profits could increase today by fishing more.

A final technical remark is that the assumption that $C_{h \alpha}^{\prime \prime}(0, x, \alpha)<0$ is crucial for the result in Proposition 5 that $\partial x_{c} / \partial \alpha$ is positive for all $\alpha$. It is however a sufficiency condition and not a necessary condition. If $C_{h \alpha}^{\prime \prime}(0, x, \alpha) \geq 0$ there may be some intervals of $\alpha$ values where $\partial x_{c} / \partial \alpha<0$. However, as may be seen from Fig. 4, if $\lim _{\alpha \rightarrow \infty} C(h, x, \alpha)=0$ then $x_{c}$ will increase, perhaps not monotonically, and eventually converge to the stock level that solves the equation $G^{\prime}(x)=\rho$. It follows that $h=\phi(x, \alpha)$ will also be reduced as $\alpha$ increases, but perhaps not monotonically.

\section{Concluding Remarks}

The present paper examines the basic nonlinear control variable biomass fishery model originating from Clark and Munro (1975), and demonstrates in a model of a pure schooling fishery the effects of improved productivity on optimal management. We build on a result in Nævdal and Skonhoft (2018) where it is shown that there is a lower bound for the fish stock below which it is optimal to set the harvest rate to zero. The results presented in the present paper indicate that improved technology imply that this lower bound should increase and that harvesting should decrease below the steady state.

These results were slightly modified by assuming that costs depend on the stock of fish. Improved technology implies a more conservative management regime is in contrast to the outcome of an unregulated fishery where improved productivity increases harvests and may destroy rents. It follows that the better the technology, the more important is it to properly manage fisheries. An important caveat to the results found in the present paper is that capital is assumed to be perfectly malleable in the sense that the capacity of the fishing fleet can be quickly adjusted (Clark et al. 1979). Squires and Vestergaard (2013) examines the impact of malleable capital and technical change when the objective is linear in harvest rate. The interaction between capital formation and unanticipated technical change when the objective is a nonlinear function of the harvest rate is to my knowledge not yet addressed in the literature and should be a subject for future research.

Jim Wilen (2000) bemoaned the lack of influence that resource economics has had on the practical regulation of the world's fisheries. Since then harvest control rules have gained traction as a management tool (Kvamsdal et al. 2016). These rules establish a relationship between the estimates of fish stocks at a given point in time and total allowable catch at that time. These conditions specify often takes the form of a lower boundary for stocks below which no harvesting should be permitted and harvesting as a linear function of the stock above that boundary harvest (Deroba and Bence 2008; Engen et al. 1997; Kvamsdal 2016). Nævdal and Skonhoft (2018) argue that this is consistent with optimal management as such a bound, termed $x_{c}$ above, appears endogenously in general models of fish management. Indeed, Fig. 5 shows that in a fairly general model, optimal harvesting as function of the stock takes exactly the shape as proposed harvest control rules. The results in the present paper show how harvest control rules that approximate optimal management policy should be changed in response to changes in technology. In general, improved technology should lead to more restrictive harvest control rules for low stock sizes. To the author's knowledge this issue has not been addressed in the literature. The results were derived within a model without cohort dynamics. However, if the cohort dynamics can be 
simplified through the use of slow-fast dynamics it was shown in Nævdal and Skonhoft (2018) that a two cohort model could be made analytically equivalent to a single equation model. The results in the present paper thus carry over to simple age structured models.

Acknowledgements The author is grateful for comments and suggestions from Michael R. Caputo, Nils Christian Framstad, Martin Quaas, William Sigurdsson, Anders Skonhoft, Stein Ivar Steinshamn, Niels Vestergaard and Jim Wilen. Funding from Norwegian Research Council's program Environment 2015, project 196199 is gratefully acknowledged.

Open Access This article is licensed under a Creative Commons Attribution 4.0 International License, which permits use, sharing, adaptation, distribution and reproduction in any medium or format, as long as you give appropriate credit to the original author(s) and the source, provide a link to the Creative Commons licence, and indicate if changes were made. The images or other third party material in this article are included in the article's Creative Commons licence, unless indicated otherwise in a credit line to the material. If material is not included in the article's Creative Commons licence and your intended use is not permitted by statutory regulation or exceeds the permitted use, you will need to obtain permission directly from the copyright holder. To view a copy of this licence, visit http://creativecommons.org/licenses/by/4.0/.

\section{References}

Bertoletti P, Rampa G (2013) On inferior inputs and marginal returns. J Econ 109:303-313

Blanchard OJ, Fischer S (1989) Lectures on macroeconomics. MIT Press, Cambridge

Brander JA, Taylor MS (1997) International trade and open-access renewable resources: the small open economy case. Can J Econ 30(3):526-552

Caputo MR (1989) The qualitative content of renewable resource models. Nat Resour Model 3(2):241-259

Clark C (1973) Profit maximization and the extinction of animal species. J Polit Econ 81:950-961

Clark C (1990) Mathematical bioeconomics. Wiley, New York

Clark C (2005) Worldwide crises in fisheries. Cambridge UP, Cambridge

Clark C, Munro GR (1975) The economics of fishing and modern capital theory: a simplified approach. J Environ Econ Manag 2(2):92-106

Clark CW, Clarke FH, Munro GR (1979) The optimal exploitation of renewable resource stocks: problems of irreversible investment. J Econ Soc 47(1):25-47

Conrad J, Clark C (1987) Natural resource economics. Notes and problems. Cambridge University Press, Cambridge

Cropper ML, Lee DR, Pannu SS (1979) The optimal extinction of a renewable natural resource. J Environ Econ Manag 6(4):341-349

Deroba J, Bence J (2008) A review of harvest policies: understanding relative performance of control rules. Fish Res 94:210-223

Engen S, Lande R, Saether BE (1997) Harvesting strategies for fluctuating populations based on uncertain population estimates. J Theor Biol 186:201-212

FAO (2018) The state of world fisheries and aquaculture 2018-meeting the sustainable development goals. Rome

Gordon D, Hannesson R (2015) The Norwegian winter herring fishery: a story of technological progress and stock collapse. Land Econ 91:362-385

Hannesson R, Salvanes KG, Squires D (2010) Technological change and the tragedy of the commons: the Lofoten fishery over 130 years. Land Econ 86(4):746-765

Judd KL (1998) Numerical methods in economics. MIT Press, Cambridge

Kvamsdal SF, Eide A, Ekerhovd N, Enberg K, Gudmundsdottir A, Hoel AH, Mills KE, Mueter FJ, Jonsen LR, Sandal LK, Stiansen JE, Vestergaard N (2016) Harvest control rules in modern fisheries management. Elem Sci Anthropocene 4:000114. https://doi.org/10.12952/journal.elementa.000114

Leung A, Wang A (1976) Analysis of models for commercial fishing: mathematical and economical aspects. Econometrica 44(2):295-303

Morey ER (1986) Allocating effort among common property cod stocks (a generalized harvest function). J Environ Econ Manag 13(1):30-49

Nævdal E, Skonhoft A (2018) New insights from the canonical fisheries model—optimal management when stocks are low. J Environ Econ Manag 92:125-133

Neher PA (1990) Natural resource economics. Cambridge University Press, Cambridge 
Skonhoft A, Quaas M (2019) Harvesting efficiency and welfare in restricted open-access fisheries. Paper presented at 24th annual conference of the european association of environmental and resource economists in Manchester in 2019

Squires D, Vestergaard N (2013) Technical change and the commons. Rev Econ Stat 95(1):1769-1787

Squires D, Vestergaard N (2018) Rethinking the commons problem: technical change, knowledge spillovers, and social learning. J Environ Econ Manag 91(3):1-25

Sydsæter K, Hammond P, Seierstad A, Strøm A (2008) Further mathematics for economic analysis, 2nd edn. Prentice Hall, Harlow

Whitmarsh D (1990) Technological change and marine fisheries development. Mar Policy 14:15-22

Wilen J (2000) Renewable resource economists and policy: what differences have we made? J Environ Econ Manag 39:306-327

World Bank (2017) The sunken billions revisited: progress and challenges in global marine fisheries. Environment and development. World Bank. (C) World Bank, Washington, DC. https://openknowledge.worldbank. org/handle/10986/24056

Publisher's Note Springer Nature remains neutral with regard to jurisdictional claims in published maps and institutional affiliations. 